Pacific Journal of Mathematic 


\title{
INVERSION AND REPRESENTATION THEOREMS FOR A GENERALIZED LAPLACE INTEGRAL
}

\author{
K. M. SAKSENA
}

1. Varma [8] introduced a generalization of the Laplace integral

$$
\mathscr{F}(x)=\int_{0}^{\infty} e^{-x t} \phi(t) d t
$$

in the form

$$
F(x)=\int_{0}^{\infty}(x t)^{m-1 / 2} e^{-x t / 2} W_{k, m}(x t) \phi(t) d t
$$

where $\phi(t) \in L(0, \infty), m>-1 / 2$ and $x>0$. This generalization is a slight variant of an equivalent integral introduced earlier by Meijer [7] and reduces to (1) when $k+m=1 / 2$. In a recent paper [1] Erdélyi has pointed out that the nucleus of (2) can be expressed as a fractional integral of $e^{-x t}$ in terms of the operators of fractional integration introduced by Kober [6]. In this note two theorems have been given-one giving an inversion formula for the transform (2) and another giving necessary and sufficient conditions for the representation of a function as an intgral of the form (2) by considering its nucleus as a fractional integral of $e^{-x t}$.

2. The operators are defined as follows.

$$
\begin{aligned}
& I_{\eta, \alpha}^{+} \mathscr{F}(x)=\frac{1}{\Gamma(\alpha)} x^{-\eta-\alpha} \int_{0}^{x}(x-u)^{\alpha-1} u^{\eta} \mathscr{F}(u) d u \\
& K_{\zeta, \alpha}^{-} \mathscr{F}(x)=\frac{1}{\Gamma(\alpha)} x^{\zeta} \int_{x}^{\infty}(u-x)^{\alpha-1} u^{-\zeta-\alpha} \mathscr{F}(u) d u
\end{aligned}
$$

where $\mathscr{F}(x) \in L_{p}(0, \infty), 1 / p+1 / q=1$ if $1<p<\infty, 1 / q=0$ if $p=1$, $\alpha>0, \eta>-1 / q, \zeta>-1 / p$.

The Mellin transform $\bar{M}_{t} \mathscr{F}(x)$ of a function $\mathscr{F}(x) \in L_{p}(0, \infty)$ is defined as

$$
\bar{M}_{t} \mathscr{F}(x)=\int_{0}^{\infty} \mathscr{F}(x) x^{i t} d x
$$

and

$$
=\lim _{X \rightarrow \infty}^{\text {index } q} \int_{1 / X}^{X} \mathscr{F}(x) x^{i t-1 / q} d x
$$

Received May 8, 1958. 
The inverse Mellin transform $\bar{M}^{-1} \phi(t)$ of a function $\phi(t) \in L_{q}(-\infty, \infty)$ is defined by

$$
\bar{M}^{-1} \phi(t)=\frac{1}{2 \pi} \int_{-\infty}^{\infty} \phi(t) x^{-i t} d t \quad(q=1)
$$

and

$$
=\frac{1}{2 \pi}-\lim _{T \rightarrow \infty}^{\text {index } p} \int_{-T}^{T} \phi(t) x^{-i t-1 / p} d t
$$

If the Mellin transform is applied to Kober's operators and the orders of integration are interchanged we obtain, under certain conditions,

$$
\bar{M}_{t}\left\{I_{\eta, \alpha}^{+} \mathscr{F}(x)\right\}=\frac{\Gamma\left(\eta+\frac{1}{q}-i t\right)}{\Gamma\left[\alpha+\left(\eta+\frac{1}{q}-i t\right)\right]} \bar{M}_{t} \mathscr{F}(x)
$$

and

$$
\bar{M}_{t}\left\{K_{\zeta, \alpha}^{-} \mathscr{F}(x)\right\}=\frac{\Gamma\left(\zeta+\frac{1}{p}+i t\right)}{\Gamma\left[\alpha+\left(\zeta+\frac{1}{p}+i t\right)\right]} \bar{M}_{t} \mathscr{F}(x) .
$$

But

$$
\bar{M}_{t}\left(e^{-x}\right)=\int_{0}^{\infty} e^{-x} x^{i t-1 / q} d x=\Gamma\left(\frac{1}{p}+i t\right) \text { if } \frac{1}{p}>0 .
$$

Therefore

$$
\bar{M}_{t}\left\{I_{\eta, \alpha}^{+}\left(e^{-x}\right)\right\}=\frac{\Gamma\left(\eta+\frac{1}{q}-i t\right) \Gamma\left(\frac{1}{p}+i t\right)}{\Gamma\left[\alpha+\left(\eta+\frac{1}{q}-i t\right)\right]}
$$

and

$$
\bar{M}_{t}\left\{K_{\zeta, \alpha}^{-}\left(e^{-x}\right)\right\}=\frac{\Gamma\left(\zeta+\frac{1}{p}+i t\right) \Gamma\left(\frac{1}{p}+i t\right)}{\Gamma\left[\alpha+\left(\zeta+\frac{1}{p}+i t\right)\right]} .
$$

By (3) 


$$
I_{\eta, \alpha}^{+}\left(e^{-x}\right)=\frac{1}{2 \pi} \int_{-\infty}^{\infty} \frac{\Gamma\left(\eta+\frac{1}{q}-i t\right) \Gamma\left(\frac{1}{p}+i t\right)}{\Gamma\left[\alpha+\left(\eta+\frac{1}{q}-i t\right)\right]} x^{-i t-1 / n} d t
$$

and

$$
K_{\zeta, \alpha}^{-}\left(e^{-x}\right)=\frac{1}{2 \pi} \int_{-\infty}^{\infty} \frac{\Gamma\left(\zeta+\frac{1}{p}+i t\right) \Gamma\left(\frac{1}{p}+i t\right)}{\Gamma\left[\alpha+\left(\zeta+\frac{1}{p}+i t\right)\right]} x^{-i t-1 / p} d t
$$

provided that $1 / p>0, \eta+1 / q>0$ and $\zeta+1 / p>0$.

It has also been shown by Erdélyi [2] that if the integral in (4) is evaluated by the calculus of residues then it can be expressed in terms of a confluent hypergeometric function. In particular,

$$
K_{2 m,(1 / 2)-m-k}^{-}\left(e^{-x}\right)=x^{m-1 / 2} e^{-x / 2} W_{k, m}(x)
$$

where $x>0,(1 / 2)-m-k>0$.

3. Theorem 1. Assume $\phi(t) \in L_{p}(0, \infty), 1 \leqq p<\infty, x>0$. If $2 m>-1 / q$ when $(1 / 2)-m-k>0$ and $(1 / 2)+m-k>-1 / q$ when $(1 / 2)-m-k>0$, then $K_{2 m,(1 / 2)-m-k}^{-}[\mathscr{F}(x)]$ exists and is equal to

$$
\int_{0}^{\infty} K_{2 m,(1 / 2)-m-k}^{-}\left(e^{-x t}\right) \phi(t) d t=F(x)
$$

where $\mathscr{F}(x)$ and $F(x)$ are given by (1) and (2) respectively.

Proof. Case I $(1 / 2)-m-k>0,1<p<\infty$.

If $\phi(t) \in L_{p}(0, \infty), 1 \leqq p<\infty$ and $x>0$ it is easy to see that $\mathscr{F}(x)$ exists. Therefore

$$
\begin{aligned}
& K_{2 m,(1 / 2)-m-k}^{-}[\mathscr{F}(x)]=\frac{x^{2 m}}{\Gamma((1 / 2)-m-k)} \\
& \quad \times \int_{x}^{\infty}(u-x)^{-(1 / 2)-m-k} u^{-(1 / 2)-m+k}\left\{\int_{0}^{\infty} e^{-u t} \phi(t) d t\right\} d u .
\end{aligned}
$$

But from a theorem of Hardy [5] we know that if $\phi(t) \in L_{p}(0, \infty)$, $1<p<\infty$ then $u^{1-2 / p} \mathscr{F}(u) \in L_{p}(0, \infty)$ and therefore $(u-x)^{\alpha} u^{\beta} \mathscr{F}(u) \in L_{p}(x, \infty)$ provided that $\alpha+\beta=1-2 / p$ and $\alpha p>-1$. Therefore the integral

$$
\begin{aligned}
& \int_{x}^{\infty}(u-x)^{-(1 / 2)-m-K} u^{-(1 / 2)-m+K} \mathscr{F}(u) d u \\
& \quad=\int_{x}^{\infty}\left\{(u-x)^{-(1 / 2)-m-k-\alpha} u^{-(1 / 2)-m+k-\beta}\right\}\left\{(u-x)^{\alpha} u^{\beta} \mathscr{F}(u)\right\} d u
\end{aligned}
$$


will exist if the expressions within the brackets in the integrand belong to $L_{p}(x, \infty)$ and $L_{q}(x, \infty)$ respectively. The conditions for these are $(-(1 / 2)-m-k-\alpha) q>-1, \quad(-1-2 m-\alpha-\beta) q<-1$ and $\alpha+\beta=1-2 / p, \alpha p>-1$ which reduce to $2 m>-1 / q$ and $(1 / 2)-m-$ $k>0$. Hence under these conditions the integral converges absolutely and we can change the order of integration. Therefore

$$
\begin{aligned}
& K_{2 m,(1 / 2)-m-K}^{-}[\mathscr{F}(x)]=\frac{x^{2 m}}{\Gamma((1 / 2)-m-k)} \int_{0}^{\infty} v^{-(1 / 2)-m-k}(x+v)^{-(1 / 2)-m+k} e^{-v t} \\
& \quad \times\left\{\int_{0}^{\infty} e^{-x t} \phi(t) d t\right\} d v=\frac{x^{2 m}}{\Gamma((1 / 2)-m-k)} \int_{0}^{\infty} e^{-x t} \phi(t) \\
& \quad \times\left\{\int_{0}^{\infty} v^{-(1 / 2)-m-k}(x+v)^{-(1 / 2)-m+k} e^{-v t} d v\right\} d t \\
& \quad=\int_{0}^{\infty}(x t)^{m-(1 / 2)} e^{-(1 / 2) x t} W_{k,-m}(x t) \phi(t) d t=F(x)
\end{aligned}
$$

as $W_{k,-m}(x)=W_{k, m}(x)$.

If $p=1$, it is similarly seen that the change in the order of integration is justified if $2 m>0$ and $(1 / 2)-m-K>0$.

Case II. $(1 / 2)-m-k<0,1<p<\infty$.

If $\alpha<0$ then the operator $K_{\eta, \alpha}^{-}\{\mathscr{F}(x)\}$ is defined as the solution, if any, of the integral equation $\mathscr{F}(x)=K_{\eta+\alpha,-\alpha}^{-}\{g(x)\}$. Now

$$
\begin{aligned}
& K_{(1 / 2)+m-k,-(1 / 2)+m+k}^{-}[F(x)] \\
& \quad=\frac{x^{(1 / 2)+m-k}}{\Gamma(-(1 / 2)+m+k)} \int_{0}^{\infty}(u-x)^{-(3 / 2)+m+k} u^{-2 m} \\
& \quad \times\left\{\int_{0}^{\infty}(u t)^{m-(1 / 2)} e^{-(1 / 2) u t} W_{K, m}(u t) \phi(t) d t\right\} d u .
\end{aligned}
$$

Again from a result of Hardy [5] we know that if

$$
F(x)=\int_{0}^{\infty} K(x y) \phi(y) d y
$$

then

$$
\int_{0}^{\infty} x^{p-2}\{F(x)\}^{p} d x<\left\{\psi\left(\frac{1}{q}\right)\right\}^{p} \int_{0}^{\infty}\{\phi(y)\}^{p} d y
$$

where

$$
\psi(s)=\int_{0}^{\infty} x^{s-1} K(x) d x
$$




$$
K(x)=\left|x^{m-(1 / 2)} e^{-(1 / 2) x} W_{k, m}(x)\right|
$$

then

$$
\psi(s)=\frac{\Gamma(2 m+s) \Gamma(s)}{\Gamma\left(m-k+\frac{1}{2}+s\right)}
$$

by Goldstein's formula [4]. Therefore

$$
\int_{0}^{\infty} x^{p-2}\{F(x)\}^{n} d x<\left\{\frac{\Gamma\left(2 m+\frac{1}{q}\right) \Gamma\left(\frac{1}{q}\right)}{\Gamma\left(m-k+\frac{1}{2}+\frac{1}{q}\right)}\right\}^{p} \int_{0}^{\infty}\{\phi(y)\}^{p} d y
$$

provided that $2 m>-1 / q$, or $x^{1-(2 / p)} F(x) \in L_{p}(0, \infty)$ if $\phi(y) \in L_{p}(0, \infty)$ $(p>1)$. Hence $(u-x)^{\alpha} u^{\beta} F(u) \in L_{p}(x, \infty)$ if $\alpha+\beta=1-(2 / p)$ and $\alpha>-1 / p$. Also $(u-x)^{-(3 / 2)+m+k-\alpha} u^{-2 m-\beta} \in L_{q}(x, \infty)$ if $(-(3 / 2)+m+k-$ $\alpha) q+1>0$ and $(-(3 / 2)-m+k-\alpha-\beta) q+1<0$. These four conditions reduce to $m+k-(1 / 2)>0$ and $m-k+(1 / 2)>-1 / q$. So the integral $\int_{x}^{\infty}(u-x)^{-(3 / 2)+m+k} u^{-2 m} F(u) d u$ exists under these conditions and

$$
\begin{aligned}
& K_{(1 / 2)+m-k,-(1 / 2)+m+k}^{-}[F(x)] \\
& \quad=\frac{x^{(1 / 2)+m-K}}{\Gamma^{\prime}(-(1 / 2)+m+k)} \int_{0}^{\infty} t^{m-(1 / 2)} \phi(t) d t \\
& \quad \times \int_{x}^{\infty}(u-x)^{m+k-(3 / 2)} u^{-m-(1 / 2)} e^{-(1 / 2) u t} W_{k, m}(u t) d u
\end{aligned}
$$

on changing the order of integration which is permissible since the integral is absolutely convergent. But [4]

$$
\int_{x}^{\infty} u^{\lambda-1}(u-x)^{k-\lambda-1} e^{-u / 2} W_{k, m}(u) d u=\Gamma(k-\lambda) x^{k-1} e^{-x / 2} W_{\lambda, m}(x)
$$

where $k>\lambda$ and $x$ is positive. Therefore

$$
\begin{aligned}
K_{(1 / 2)+m-k,-(1 / 2)+m+k}^{-}[F(x)] & =\int_{0}^{\infty}(x t)^{m-(1 / 2)} e^{-(x t / 2)} W_{-m+(1 / 2), m}(x t) \phi(t) d t \\
& =\int_{0}^{\infty} e^{-x t} \phi(t) d t
\end{aligned}
$$

under the conditions $m+k-(1 / 2)>0, m-k+(1 / 2)>-1 / q, x>0$.

If $p=1$, the change in the order of integration is justified if $m+K-(1 / 2)>0$ and $(1 / 2)+m-k>0$.

Hence $K_{(1,2)+m-k,-(1 / 2)+m+k}^{-}[F(x)]=\mathscr{F}(x)$ and the theorem is proved.

THEOREM 2. Under the conditions of Theorem 1 we have 


$$
\int_{0}^{\infty} e^{-x t} I_{2 m,(1 / 2)-m-K}^{+}\{\phi(t)\} d t=\int_{0}^{\infty} K_{2 m,(1 / 2)-m-K}^{-}\left(e^{-x t}\right) \phi(t) d t .
$$

This is a consequence of Theorem 2 of Erdélyi [3] and is proved similarly.

4. We are now in a position to give inversion and representation theorems for the transform.

We have seen that, under certain conditions,

$$
K_{(1 / 2)+m-k,-(1 / 2)+m+k}^{-}[F(x)]=\mathscr{F}(x) .
$$

Also $\mathscr{F}(x)$ has derivatives of all orders for $x$ sufficiently large and vanishes at infinity. So we can apply the Post-Widder operator $L_{\lambda, u}$ defined by the relation

$$
L_{\lambda, u}\left[\mathscr{F}^{(x)}\right]=\frac{(-1)^{\lambda}}{\lambda !} \mathscr{F}^{(\lambda)}\left(\frac{\lambda}{u}\right)\left(\frac{\lambda}{u}\right)^{\lambda+1}
$$

(where $\lambda$ is a positive integer and $u$ a real positive number) to $\mathscr{F}(x)$ and obtain an inversion theorem.

Lemma. If $\phi(t) \in L_{p}$ in $(0 \leqq t<\infty)$ and

$$
\psi(u)=\int_{0}^{\infty}|\phi(u t)-\phi(t)|^{p} d t
$$

then

$$
\left|\frac{u \psi(u)}{1+u}\right| \leqq\|\phi\|_{p}^{p} \text { for } u \geqq 0
$$

and

$$
\psi(u) \rightarrow 0 \text { as } u \rightarrow 1
$$

where $\|\mathscr{F}\|_{p}$ denotes the norm of the function $\mathscr{F}(t) \in L_{p}(0, \infty)$, that is

$$
\|\mathscr{F}\|_{p}=\left\{\int_{0}^{\infty}|\mathscr{F}(t)|^{p} d t\right\}^{(1 / p)} .
$$

Proof. We have

$$
|\psi(u)| \leqq \int_{0}^{\infty}|\phi(u t)|^{p} d t+\int_{0}^{\infty}|\phi(t)|^{p} d t=\left(1+\frac{1}{u}\right) \int_{0}^{\infty}|\phi(t)|^{p} d t
$$

which proves (i).

Also, by a change of variable, 


$$
\phi\left(e^{y}\right)=\int_{-\infty}^{\infty}\left|\phi\left(e^{x+y}\right)-\phi\left(e^{x}\right)\right|^{p} e^{x} d x
$$

If $\alpha(x)=e^{(x / p)} \phi\left(e^{x}\right)$ then

$$
\int_{-\infty}^{\infty}|\alpha(x)|^{p} d x=\int_{-\infty}^{\infty}\left|\phi\left(e^{x}\right)\right|^{p} e^{x} d x=\|\phi\|_{p}^{p}
$$

and so $\alpha(x) \in L_{p}(-\infty, \infty)$. Again

$$
\begin{aligned}
\left\{\psi\left(e^{y}\right)\right\}^{1 / p} & =\left[\int_{-\infty}^{\infty} \mid\left\{\alpha(x+y) e^{-(y / p)}-\alpha(x) e^{-(y / p)}\right\}\right. \\
& \left.+\left.\left\{\alpha(x) e^{-(y / p)}-\alpha(x)\right\}\right|^{p} d x\right]^{1 / p} \\
& \leqq e^{-(y / p)}\left[\int_{-\infty}^{\infty}|\alpha(x+y)-\alpha(x)|^{p} d x\right]^{1 / p} \\
& +\left|e^{-(y / p)}-1\right|\left[\int_{-\infty}^{\infty}|\alpha(x)|^{p} d x\right]^{1 / p}
\end{aligned}
$$

by Minkowski's inequality. And $\int_{-\infty}^{\infty}|\alpha(x+y)-\alpha(x)|^{p} d x \rightarrow 0$ as $y \rightarrow 0$ if $\alpha(x) \in L_{p}(-\infty, \infty)$ and so does $\left|e^{-y / p}-1\right|$. Therefore $\psi\left(e^{y}\right)=o$ (1) as $y \rightarrow 0$ or $\phi(u) \rightarrow 0$ as $u \rightarrow 1$.

THEOREM 3. Assume $\phi(t) \in L_{p}(1 \leqq p<\infty)$ in $0 \leqq t \leqq R$ for every positive $R$. If the integral $\mathscr{F}(x)$ converges for $x>0$ and $2 m>-1 / q$ when $(1 / 2)-m-k>0 ;(1 / 2)+m-k>-1 / q$ when $(1 / 2)-m-k<0$, then, for almost all positive $t$,

$$
\underset{\lambda \rightarrow \infty}{\operatorname{index} p} L_{\lambda, t}\left[K_{(1 / 2)+m-k,-(1 / 2)+m+k}^{-}\{F(x)\}\right]=\phi(t) \text {. }
$$

Proof. We have seen in the proof of Theorem 1 that, under the conditions of the theorem,

$$
K_{(1 / 2)+m-k,-(1 / 2)+m+k}\{F(x)\}=\mathscr{F}(x) .
$$

Therefore

$$
\begin{aligned}
L_{\lambda, t} & \equiv L_{\lambda, t}\left[K_{(1 / 2)+m-k,-(1 / 2)+m+k}\{F(x)\}\right] \\
& =\frac{1}{\lambda !}\left(\frac{\lambda}{t}\right)^{\lambda+1} \int_{0}^{\infty} e^{-(\lambda u / t)} u^{\lambda} \phi(u) d u
\end{aligned}
$$

by simple computation and

$$
\begin{aligned}
\left|L_{\lambda, t}-\phi(t)\right| & \leqq \frac{1}{\lambda !}\left(-\frac{\lambda}{t}\right)^{\lambda+1} \int_{0}^{\infty} e^{-(\lambda u / t)} u^{\lambda}|\phi(u)-\phi(t)| d u \\
& =\frac{1}{\lambda !} \lambda^{\lambda+1} \int_{0}^{\infty} e^{-\lambda v} v^{\lambda}|\phi(v t)-\phi(t)| d v .
\end{aligned}
$$


Therefore

$$
\begin{aligned}
\left|L_{\lambda, t}-\phi(t)\right|^{p} \leqq & \left|\frac{\lambda^{\lambda+1}}{\lambda !} \int_{0}^{\infty} e^{-\lambda v} v^{\lambda}\right| \phi(v t)-\phi(t)|d v|^{p} \\
\leqq & {\left[\frac{\lambda^{\lambda+1}}{\lambda !} \int_{0}^{\infty} e^{-\lambda v} v^{\lambda}|\phi(v t)-\phi(t)|^{p} d v\right]\left[\frac{\lambda^{\lambda+1}}{\lambda !} \int_{0}^{\infty} e^{-\lambda v} v^{\lambda} d v\right]^{p / q} } \\
& \frac{\lambda^{\lambda+1}}{\lambda !} \int_{0}^{\infty} e^{-\lambda v} v^{\lambda}|\phi(v t)-\phi(t)|^{p} d v .
\end{aligned}
$$

Hence

$$
\begin{aligned}
\int_{0}^{\infty}\left|L_{\lambda, t}-\phi(t)\right|^{p} d t & \leqq \frac{\lambda^{\lambda+1}}{\lambda !} \int_{0}^{\infty} d t \int_{0}^{\infty} e^{-\lambda v} v^{\lambda}|\phi(v t)-\phi(t)|^{p} d v \\
& =\frac{\lambda^{\lambda+1}}{\lambda !} \int_{0}^{\infty} e^{-\lambda v} v^{\lambda} d v\left\{\int_{0}^{\infty}|\phi(v t)-\phi(t)|^{p} d t\right\} .
\end{aligned}
$$

In changing the order of integration, this becomes

$$
\frac{\lambda^{\lambda+1}}{\lambda !} \int_{0}^{\infty} e^{-\lambda v} v^{\lambda} \psi(v) d v
$$

where $\psi(v)$ is defined as in the lemma. From the lemma it is easily seen that

$$
\begin{aligned}
\psi(u) & =0(1) & & (u \rightarrow \infty) \\
& =0\left(u^{-1}\right) & & (u \rightarrow 0+)
\end{aligned}
$$

Therefore $\int_{0}^{\infty} e^{-\lambda v} v^{\lambda} \psi(v) d v$ converges for $\lambda \geqq 1$ and the inversion of the order of integration is justified by Fubini's theorem. By a familiar result [9, Theorem 3c, p. 283] the integral (6) approaches $\psi(1)$ as $\lambda \rightarrow \infty$. But, by the lemma, $\psi(u)=o(1)$ as $u \rightarrow 1$. Therefore $L_{\lambda, t}$ converges in mean to $\phi(t)$ with index $p$ on $0 \leqq t<\infty$ and the result is proved.

THEOREM 4. The necessary and sufficient conditions for a function $F(x)$ to have the representation (2) with $\phi(t) \in L_{p}(0, \infty), p \geqq 1, x>1$, and with $2 m>-1 / q$ when $1 / 2-m-K>0$ and $m-k+1 / 2>-1 / q$ when $1 / 2-m-k<0$ are

(i) $K_{1 / 2+m-K,-1 / 2+m+K}^{-}\{F(x)\} \equiv G(x)$ exists, has derivatives of all orders in $0<x<\infty$ and vanishes at infinity and

(ii) there exist constants $M$ and $p(p \geqq 1)$ such that

$$
\int_{0}^{\infty}\left|L_{\lambda, t}[G(x)]\right|^{p} d t<M \quad(\lambda=1,2, \cdots) .
$$

Proof. First let $F(x)$ have the representation (2). Then, from Theorem 1, 


$$
G(x) \equiv K_{1 / 2+m-k,-1 / 2+m+k}^{-}\{F(x)\}=\mathscr{F}(x)
$$

and as in the proof of Widder [9, Theorem 15a, pp. 313-14] we see that the conditions are satisfied.

Conversely, let the conditions be satisfied. Then again, as in the proof of Widder's theorem referred to before, we see that

$$
G(x)=\int_{0}^{\infty} e^{-x t} \phi(t) d t=\mathscr{F}(x) .
$$

Therefore [3, p. 300]

$$
\begin{aligned}
F(x) & =\left(K_{(1 / 2)+m-k,-(1 / 2)+m+k}^{-}\right)^{-1} \mathscr{F}(x)=K_{2 m, 1 / 2-m-k}^{-}\{\mathscr{F}(x)\} \\
& =\int_{0}^{\infty}(x t)^{m-1 / 2} e^{-x t / 2} W_{K, m}(x t) \phi(t) d t
\end{aligned}
$$

by Theorem 1 ; and the theorem is proved.

CoRollary. If the fractional derivatives or integrals

$$
K_{(1 / 2)+m-k+r,-(1 / 2)+m+k-r}^{-}\{F(x)\}
$$

exist for $r=0$ and every positive integer, then the integral in the condition (ii) of Theorem 4 can be replaced by

$$
\int_{0}^{\infty} \frac{(-1)^{\lambda}}{\lambda !}\left(\frac{\lambda}{t}\right) \sum_{r=0}^{\lambda}(-1)^{r} A_{r} K_{(1 / 2)+m-k+r,(1 / 2)+m+k-r}^{-}\left\{F\left(\frac{\lambda}{t}\right)\right\}^{\prime p} d t
$$

where

$$
\begin{gathered}
A_{r}={ }^{\lambda} C_{r}(m-k+(1 / 2))(m-k-(1 / 2)) \cdots(m-k-\lambda+(3 / 2)+r) \\
(r=0,1, \cdots, \lambda-1), \quad A_{\lambda}=1 .
\end{gathered}
$$

For [6]

$$
t^{a} K_{\bar{\zeta}, \alpha}\{\mathscr{F}(t)\}=K_{\zeta+a \alpha}^{-}\left\{t^{a} \mathscr{F}(t) .\right.
$$

Therefore

$$
K_{\zeta, \alpha}^{-}\{F(x)\}=x^{\zeta} K_{0, x}^{-}\left\{x^{-\zeta} F(x)\right\}
$$

and

$$
\begin{aligned}
& \frac{d^{\lambda}}{d x^{\lambda}}\left[K_{\bar{\zeta}, \alpha}\{F(x)\}\right]=\frac{d^{\lambda}}{d x^{\lambda}}\left(x^{\zeta}\right)\left[K_{0, \alpha}^{-}\left\{x^{-\zeta} F(x)\right\}\right] \\
& \quad+{ }^{\lambda} C_{1} \frac{d^{\lambda-1}}{d x^{\lambda-1}}\left(x^{\zeta}\right) \frac{d}{d x}\left[K_{0, \alpha}^{-}\left\{x^{-\zeta} F(x)\right\}\right]+\cdots \\
& \quad+{ }^{\lambda} C_{\lambda-1} \frac{d}{d x}\left(x^{\zeta}\right) \frac{d^{\lambda-1}}{d x^{\lambda-1}}\left[K_{0, \alpha}^{-}\left\{x^{-\zeta} F(x)\right\}\right] \\
& \quad+x^{\zeta} \frac{d^{\lambda}}{d x^{\lambda}}\left[K_{0, \alpha}^{-}\left\{x^{-\zeta} F(x)\right\}\right] .
\end{aligned}
$$


By Leibnitz's theorem this becomes

$$
\begin{aligned}
& =\zeta(\zeta-1) \cdots(\zeta-\lambda+1) x^{\zeta-\lambda}\left[K_{0, \alpha}^{-}\left\{x^{-\zeta} F(x)\right\}\right] \\
& -{ }^{\lambda} C_{1} \zeta(\zeta-1) \cdots(\zeta-\lambda+2) x^{\zeta-\lambda+1}\left[K_{0, \alpha-1}^{-}\left\{x^{-\zeta-1} F(x)\right\}\right] \\
& +\cdots+(-1)^{\lambda} x^{\zeta}\left[K_{0, \alpha-\lambda}^{-}\left\{x^{-\zeta-\lambda} F(x)\right] .\right.
\end{aligned}
$$

Therefore

$$
\begin{aligned}
& \frac{(-1)^{\lambda}}{\lambda^{1}} x^{\lambda+1} \frac{d^{\lambda}}{d x^{\lambda}}\left[K_{\zeta, \alpha}^{-}\{F(x)\}\right] \\
& =\frac{(-1)^{\lambda}}{\lambda !} \sum_{r=0}^{\lambda}(-1)^{r} A_{r} x^{\zeta+r+1}\left[K_{0, \alpha-r}^{-}\left\{x^{-\zeta-r} F(x)\right\}\right]
\end{aligned}
$$

where

$$
\begin{aligned}
& A_{r}={ }^{\lambda} C_{r} \zeta(\zeta-1) \cdots(\zeta-\lambda+r+1) \\
& A_{\lambda}=1, \quad(r=0,1, \cdots, \lambda-1),
\end{aligned}
$$

and

$$
\begin{aligned}
L_{\lambda, t}\left[K_{\overline{\zeta, \alpha}}\{F(x)\}\right] & =\frac{(-1)^{\lambda}}{\lambda !} \sum_{r=0}^{\lambda}(-1)^{r} A_{r}\left(\frac{\lambda}{t}\right)^{\zeta+r+1}\left[K_{0, \alpha-r}^{-}\left\{\left(\frac{\lambda}{t}\right)^{-\zeta-r} F\left(\frac{\lambda}{t}\right)\right\}\right] \\
& =\frac{(-1)^{\lambda}}{\lambda !}\left(\frac{\lambda}{t}\right) \sum_{r=0}^{\lambda}(-1)^{r} A_{r}\left[K_{\bar{\zeta}+r, \alpha-r}\left\{F\left(\frac{\lambda}{t}\right)\right\}\right] .
\end{aligned}
$$

Putting $\zeta=m-k+1 / 2$ and $\alpha=m+k-1 / 2$ we have the required result.

THEOREM 5a. If $F(x)$ has representation (2) with the conditions of Theorem 4 on $\phi(t), x, k$ and $m$ satisfied and if the fractional derivatives or integrals $K_{(1 / 2)+m-k+r,-(1 / 2)+m+k-r}^{-}\{F(x)\}$ exist for $r=0$ and every positive integer, than

$$
\lim _{\lambda \rightarrow \infty} \int_{0}^{\infty}\left|\frac{(-1)^{\lambda}}{\lambda !}\left(\frac{\lambda}{t}\right) \sum_{r=0}^{\lambda}(-1)^{r} A_{r}\left[K_{(1 / 2)+m-k+r,(-1 / 2)+m+k-r}^{-}\left\{F\left(\frac{\lambda}{t}\right)\right\}\right]\right|^{p} d t=\|\phi\|_{p}^{p} .
$$

where the $A_{r}$ 's have values as in the Corollary to Theorem 4.

Proof. The proof is similar to that of Widder [9, Theorem 15b, p. 314]

THEOREM 5b. If the function $F(x)$ has representation (2) with the conditions of Theorem 4 on $\phi(t), x, k$ and $m$ satisfied, then

$$
\left.\lim _{\lambda \rightarrow \infty} \int_{0}^{\infty}\left|L_{\lambda, t}\{F(x)\}^{p} d t=\int_{0}^{\infty}\right| I_{2 m,(1 / 2)-m-k}^{+}\{\phi(t)\}\right|^{p} d t .
$$

Proof. If $F(x)$ has the representation (2), then, by Theorem 2 we have 


$$
F(x)=\int_{0}^{\infty} e^{-x t} I_{2 m,(1 / 2)-m-k}^{+}\{\phi(t)\} d t .
$$

Also if $\phi(t) \varepsilon L_{p}(0, \infty)$ so does $I_{2 m,(1 / 2)-m-k}^{+}\{\phi(t)\}$ provided that $2 m>-1 / q$. Therefore, as in Widder [9, Theorem 15b, p. 314], we can prove again that

$$
\lim _{\lambda \rightarrow \infty} \int_{0}^{\infty}\left|L_{\lambda, t}\{F(x)\}\right|^{p} d t=\int^{\infty} \mid I_{2 m,(1 / 2)-m-k}^{+}\{\phi(t)\}^{p} d t
$$

I am deeply grateful to Professor A. Erdélyi for many helpful suggestions.

\section{REFERENCES}

1. A. Erdélyi, On a generalization of the Laplace transformation, Proc. Edin. Math. Soc., Ser. (2) 10 (1951), 53-55.

2. ___- On some functional transformations, Rend. del Semin. Mat. 10 (1950-51) 217-234.

3. - On fractional integration and its application to the theory of Hankel transforms, Quart. J. Math. 11, (1940), 293-303.

4. S. Goldstein, Operational representations of Whittaker's confluent hypergeometric function and Weber's parabolic cylinder function, Proc. Lond. Math. Soc., (2) 34 (1932), 103-125.

5. G. H. Hardy, The constants of certain inequalities, J. Lond. Math.Soc., 8, (1933), 114-211.

6. H. Kober, On fractional integrals and derivatives, Quart. Jour. Math., 11, (1940), 193-211.

7. C. S. Meijer, Eine noue Erweiterung der Laplace Transformation, I, Proc, Sect, Sci., Amsterdam Akad. Wet. 44, (1941), 727-737.

8. R. S. Varma, On a generalization of Laplace integral, Proc. Nat. Acad. Sci. (India), A 20, (1951), 209-216.

9. D. V. Widder, The Laplace transform, 1941.

California Institute of Technology

D. S. B. Government College, Naini TaI, India. 



\section{PACIFIC JOURNAL OF MATHEMATICS}

\section{EDITORS}

David Gilbarg

Stanford University

Stanford, California

R. A. Beaumont

University of Washington

Seattle 5, Washington

\author{
A. L. Whiteman
}

University of Southern California

Los Angeles 7, California

E. G. Straus

University of California

Los Angeles 24, California

\section{ASSOCIATE EDITORS}

\author{
E. F. BECKENBACH \\ C. E. BURGESS \\ M. HALL \\ E. HEWITT
}

\author{
A. HORN \\ V. GANAPATHY IYER \\ R. D. JAMES \\ M. S. KNEBELMAN
}

L. NACHBIN
I. NIVEN
T. G. OSTROM
H. L. ROYDEN

M. M. SCHIFFER

G. SZEKERES

F. WOLF

K. YOSIDA

\section{SUPPORTING INSTITUTIONS}

\author{
UNIVERSITY OF BRITISH COLUMBIA \\ CALIFORNIA INSTITUTE OF TECHNOLOGY \\ UNIVERSITY OF CALIFORNIA \\ MONTANA STATE UNIVERSITY \\ UNIVERSITY OF NEVADA \\ OREGON STATE COLLEGE \\ UNIVERSITY OF OREGON \\ UNIVERSITY OF SOUTHERN CALIFORNIA
}

\author{
STANFORD UNIVERSITY \\ UNIVERSITY OF UTAH \\ WASHINGTON STATE COLLEGE \\ UNIVERSITY OF WASHINGTON \\ AMERICAN MATHEMATICAL SOCIETY \\ CALIFORNIA RESEARCH CORPORATION \\ HUGHES AIRCRAFT COMPANY \\ THE RAMO-WOOLDRIDGE CORPORATION
}

Mathematical papers intended for publication in the Pacific Journal of Mathematics should be typewritten (double spaced), and the author should keep a complete copy. Manuscripts may be sent to any of the editors. All other communications to the editors should be addressed to the managing editor, E. G. Straus at the University of California, Los Angeles 24, California.

50 reprints per author of each article are furnished free of charge; additional copies may be obtained at cost in multiples of 50 .

The Pacific Journal of Mathematics is published quarterly, in March, June, September, and December. The price per volume (4 numbers) is $\$ 12.00$; single issues, $\$ 3.50$. Back numbers are available. Special price to individual faculty members of supporting institutions and to individual members of the American Mathematical Society: $\$ 4.00$ per volume; single issues, $\$ 1.25$.

Subscriptions, orders for back numbers, and changes of address should be sent to Pacific Journal of Mathematics, 2120 Oxford Street, Berkeley 4, California.

Printed at Kokusai Bunken Insatsusha (International Academic Printing Co., Ltd.), No. 6, 2-chome, Fujimi-cho, Chiyoda-ku, Tokyo, Japan.

\section{PUBLISHED BY PACIFIC JOURNAL OF MATHEMATICS, A NON-PROFIT CORPORATION}

The Supporting Institutions listed above contribute to the cost of publication of this Journal, but they are not owners or publishers and have no responsibility for its content or policies. 


\section{Pacific Journal of Mathematics}

\section{Vol. 8, No. 3 \\ May, 1958}

Michael Israel Aissen, A set function defined for convex plane domaines... . 383

Robert Ellis, Distal transformation groups ................... 401

Ciprian Foias, On a commutative extension of a commutative Banach algebra ....................................... 407

Jerry William Gaddum, Linear inequalities and quadratic forms ......... 411

Allen A. Goldstein and Elliott Ward Cheney, Jr., A finite algorithm for the solution of consistent linear equations and inequalities and for the Tchebycheff approximation of inconsistent linear equations...........

William L. Hart and T. S. Motzkin, Proof of the fundamental theorem on implicit functions by use of composite gradient corrections .......... 429

Henry Berge Helson, Conjugate series and a theorem of Paley .......... 437

Wu-Chung Hsiang, Abelian groups characterized by their independent subsets....................................... 447

John W. Lamperti, On the isometries of certain function-spaces ........ 459

Karel DeLeeuw and Walter Rudin, Extreme points and extremum problems

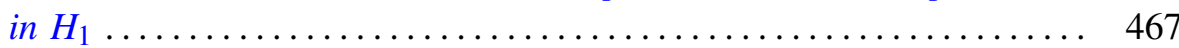

Eugene Lukacs, Some extensions of a theorem of Marcinkiewicz........ 487

George W. Mackey, Multiplicity free representations of finite groups ..... 503

Eben Matlis, Injective modules over Noetherian rings ............. 511

John William Neuberger, Continuous products and nonlinear integral equations

Lawrence Edward Payne and Hans F. Weinberger, New bounds for solutions of second order elliptic partial differential equations...

William T. Reid, A Prüfer transformation for differential systems ........ 575

Howard L. Rolf, The free lattice generated by a set of chains ...

K. M. Saksena, Inversion and representation theorems for a generalized

Laplace integral....................................... 597

Daniel Shanks, Two theorems of Gauss......................... 609

Paul Slepian, On the Lebesgue area of a doubled map ............... 613

Otto Szász and Nelson Paul Yeardley, Jr., The representation of an analytic function by general Laguerre series . ..................... 621

Alan C. Woods, On two-dimensional convex bodies ................. 635 